\title{
FÚTBOL, PRENSA E IDENTIDADES NACIONALES EN ESPAÑA, 1975-2017
}

FUTEBOL, IMPRENSA E IDENTIDADES NACIONAIS EM ESPANHA, 1975-2017

FOOTBALL, NEWSPAPERS AND NATIONAL IDENTITIES IN SPAIN, 1975-2017

Juan Antonio Simón*

\begin{abstract}
Palabras clave:
Fútbol.

Construcción Social

de la Identidad.

España.

Prensa.

Resumen: El objetivo de esta investigación fue analizar el papel que ha representado el fútbol como medio para la construcción, configuración y fortalecimiento de las identidades nacionales en España entre 1975 y 2017. A nivel metodológico, se revisó la bibliografía y las principales revistas y periódicos españoles, tanto deportivos como de información general de este periodo. Los principales resultados obtenidos nos muestran cómo la prensa ha reescrito las diversas narrativas sobre la identidad nacional española desde los últimos años, convirtiéndose en la herramienta ideal con la que conseguir asociar ciertos estereotipos y mitos con la selección española y los principales clubes de fútbol. Del mismo modo, la relación entre fútbol y medios de comunicación también ha representado un papel clave para la creación y transformación de los discursos identitarios en Cataluña y el País Vasco durante las últimas décadas.
\end{abstract}

Palavras chave

Futebol.

Construção social

da identidade.

Espanha.

Imprensa.

Keywords:

Soccer.

Social Construction

of Identity.

Spain.

Press.
Resumo: 0 objetivo desta pesquisa foi analisar o papel desempenhado pelo futebol como meio para a construção, configuração e fortalecimento das identidades nacionais na Espanha entre 1975 e 2017. No âmbito metodológico, revisaram-se a bibliografia e as principais revistas e jornais espanhóis, tanto de esportes quanto de informação geral desse período. Os principais resultados obtidos nos mostram como a imprensa reescreveu as várias narrativas sobre a identidade nacional espanhola desde os últimos anos, tornandose a ferramenta ideal para associar certos estereótipos e mitos à seleção espanhola e aos principais clubes de futebol. Do mesmo modo, a relação entre o futebol e a imprensa também desempenhou um papel fundamental na criação e transformação dos discursos identitários na Catalunha e no País Basco durante as últimas décadas.

Abstract: This research investigates the role played by football as a means to build, configure and strengthen national identities in Spain between 1975 and 2017. At the methodological level, bibliography and the main Spanish magazines and newspapers of the period were reviewed - both those featuring sports and general information. The main findings show us how the press has rewritten the various narratives about Spanish national identity in recent years and became the ideal tool to associate certain stereotypes and myths with the Spanish national football team and the main football clubs. Likewise, the relationship between football and the media has also played a key role in creating and changing identity discourses in Catalonia and the Basque Country in recent decades.
* Universidad Europea de Madrid. Faculty of Sport Sciences. Madrid, España.

E-mail: jasimonsanjurjo@ hotmail.com

Recebido em: 23/03/2018 Aprovado em: 09/11/2018

DOI: hitps://doi.org/10.22456/1982-8918.81403 (c) (i) () Licence 


\section{INTRODUCCIÓN}

El pasado 27 de octubre del 2017, el club catalán recién ascendido a primera división Girona FC, se impuso de forma inesperada en su propio estadio por 2-1 al Real Madrid CF. Sólo un día antes, el Parlamento de Cataluña había realizado la denominada Declaración Unilateral de Independencia (DUI), e inmediatamente después, el Gobierno inició la activación del artículo 155 de la constitución española, que les permitía tomar el control de la Generalitat de Cataluña y convocar nuevas elecciones para el 21 de diciembre de ese mismo año (MATEO, 2017). El domingo 29 de octubre a las once de la mañana, Carles Puigdemont, recién cesado de su cargo de presidente de la Generalitat de Cataluña al amparo de la Orden PRA/1034/2017 (BOLETíN OFICIAL DEL ESTADO, 2017), escribía en su cuenta personal de Twitter. "La victoria del @ GironaFC sobre un dels grans equips del món és tot un exemple i un referent per a moltes situacions" (PUIGDEMONT, 2017)'.

Semanas antes, concretamente el 7 de octubre, Javier Tebas Medrano, presidente de la Liga de Fútbol Profesional (LFP), también utilizaba las redes sociales para mostrar una imagen de la manifestación organizada por la Fundación DENAES, para la Defensa de la Nación Española, y en la que añadía el siguiente mensaje: "Hoy estuvimos en la plaza Colón, mañana en Barcelona, plaza Urquinaona a las 12h. Porque Cataluña es España", dejando de esta forma muy claro su posicionamiento respecto a los acontecimientos políticos que se estaban desarrollando durante esas semanas (TEBAS, 2017). Estos dos hechos, ejemplifican claramente la importancia que hoy en día representa el fútbol en la sociedad española y su reiterada utilización dentro del debate político. Su presencia constante en los medios de comunicación ayuda a generar un conjunto de interrelaciones entre fútbol, política, economía e identidades, que se han ido constatando con mayor o menor intensidad desde principios del siglo XX con el inicio de la transformación del fútbol en un espectáculo de masas.

Este artículo, tiene como objetivo principal el análisis de la utilización del fútbol en España como instrumento para dar forma a narrativas nacionales, tanto por parte de los gobiernos centrales como autonómicos, así como su plasmación en los medios de comunicación. El marco cronológico abarca desde el periodo comprendido entre el inicio de la transición a la democracia en 1975, hasta la llegada al poder de Mariano Rajoy a finales del 2011 y la situación que ha vivido el país durante el 2017. Esta investigación afronta el estudio de la evolución de los discursos sobre España a través del fútbol, así como el análisis de las diversas narrativas que desde 1975 han estado compitiendo en Cataluña y el País Vasco. En los últimos años, han sido varios los autores que han tratado desde las ciencias sociales y la historia, las interrelaciones que han existido en España entre fútbol, economía, política e identidad, pudiendo destacar entre otros los trabajos de Ramon Llopis-Goig (2015), Alejandro Quiroga (2014), González Ramallal (2014) o Carlos García (2016).

En cuanto a la estructura de este trabajo, el primer apartado analiza un periodo clave para la historia contemporánea de España como fueron los años que abarcan desde la muerte del dictador Francisco Franco en noviembre de 1975, hasta la llegada al gobierno del partido socialista en octubre de 1982. Se describe en este apartado el proceso de democratización del deporte, así como el crecimiento de los regionalismos y los nacionalismos catalanes y vascos. A continuación, se explorarán las identidades nacionales españolas y su relación con el fútbol

1 Traducción nuestra: "La victoria del @ GironaFC sobre un de los grandes equipos del mundo es todo un ejemplo y un referente para muchas situaciones". 
durante los Gobiernos de Felipe González y José María Aznar. Se ha pretendido subrayar la evolución del estereotipo de la "furia española" y la "narrativa de la mala suerte" o el fracaso, que parecía ir vinculado desde el franquismo a la selección nacional, así como la amplia cobertura que desde los medios de comunicación han recibido este tipo de discursos. En el último apartado se analiza en primer lugar la transformación de los estereotipos españoles asociados a los malos resultados y al atraso, frente a la imagen de una nueva España moderna y globalizada que vendrá asociada al nuevo estilo de fútbol técnico, sofisticado y espectacular que impondrá el FC Barcelona y la propia selección con sus triunfos en la Eurocopa del 2008 y 2012, y sobre todo en la Copa del Mundo del 2010. El artículo concluirá con la exposición del proceso que se ha intensificado desde el 2008, coincidiendo con el inicio de la crisis económica, de la reclamación de independencia desde diversos sectores en Cataluña y su relación con el fútbol.

\section{LA TRANSICIÓN DEMOCRÁTICA Y EL PROCESO DE MODERNIZACIÓN DE LA NARRATIVA DE LA FURIA Y EL FRACASO, 1975-1982}

La muerte de Franco el 20 de noviembre de 1975 y las elecciones del 15 de junio de 1977 abrieron una fase de democratización y un periodo de profunda transformación en el fútbol español, que se desarrolló de forma paralela al proceso de reforma que vivió este país. El desmantelamiento de la política deportiva del franquismo se inició con la transformación de la Delegación Nacional de Deportes (DND) en la Dirección General de Deportes y, posteriormente en agosto de 1977, en el actual Consejo Superior de Deportes (CSD), un organismo autónomo a través del cual el Estado intervendrá en el deporte (BODIN, 2011).

El fútbol también inició a partir de este momento un lento proceso de democratización institucional. El presidente del CSD, Benito Castejón, incorporó la elección democrática de los presidentes de las diferentes federaciones deportivas. Un proceso similar, también se trató de impulsar para la elección de los presidentes de las federaciones regionales de fútbol, y para nombrar al máximo representante de la Real Federación Española de Fútbol (RFEF). Pablo Porta, como presidente de esta institución, aprobó en julio de 1977 la elección de los directivos de los clubes de fútbol por sufragio universal, pese a que las reformas democráticas en el mundo del fútbol no siempre se implementaron a la velocidad que la sociedad española deseaba.

Por otro lado, desde la segunda mitad de los años setenta se apreció un considerable incremento de las demandas de mayor autonomía regional en relación con el gobierno central, que terminaron tomando forma con el modelo de organización territorial del Estado de las Autonomías. Este conjunto de reivindicaciones, encontraron en el fútbol un escaparate ideal en el que poder plasmar los nuevos discursos identitarios. Ejemplo de lo anterior, será la decisión de los capitanes de la Real Sociedad y del Athletic Club de Bilbao, Inaxio Kortabarria e Jose Ángel Iribar, de entrar en el estadio de Atocha el 6 de diciembre de 1976 junto con los demás compañeros de sus respectivos equipos, portando la bandera vasca, la ikurriña, para reclamar de esta forma su legalización (RODRIGÁLVAREZ, 2011). El periódico de extrema derecha El Alcázar, bajo el titular de "Intolerable orgía separatista", acusó a Iribar de ser "el gran culpable de todo. Iribar a quien toda España ha engrandecido, ahora se las apaña para lanzar odio contra España" (GALLO, 1976). 
Otros ejemplos de la tensión social que se vivió en España durante los primeros años de la transición, fueron hechos como la retirada de una ikurriña que portaban varios aficionados del Osasuna en su visita al campo del Racing de Santander, y que ante "[...] las protestas de buena, parte del público" se les decidió retirar (SANTANDER, 1979, p. 6); o la sanción de medio millón de pesetas que el gobernador de la provincia de Huelva impuso al jugador José María Zuloaga, por supuestamente "[...] hacer ejercicios de calentamiento [...] cuando se interpretaba el himno nacional español en conmemoración por el tercer aniversario de la Constitución", considerándolo una actitud "despectiva y de rechazo al acto", y pese a que el jugador declaró que se consideraba "tan español como el que más. Soy leal al Rey, a la Constitución y a cuantos símbolos puedan representar a España" (CABALLERO, 1981). ${ }^{2}$

Especial atención merece el análisis del papel representado por el FC Barcelona, club que se convirtió para muchos aficionados durante el periodo de la transición en un instrumento de difusión del catalanismo. Johan Cruyff, estrella del equipo y capitán en esos años, decidió llevar el brazalete con los colores de la bandera de Cataluña, la senyera, en un partido disputado el 1 de febrero de 1976 en el Camp Nou frente al Athletic Club de Bilbao. Un gesto de provocación que tendrá su continuación un año más tarde, cuando aficionados de ambos equipos desfilaron nuevamente en el Camp Nou portando señeras e ikurriñas en los prolegómenos del partido que disputaron ambos equipos (SOTERAS, 1977, p. 47). Pocos días antes, la relación entre fútbol y política ya había ocupado el primer plano de la actualidad por la reacción de los aficionados del FC Barcelona cuando Cruyff fue expulsado por segunda vez desde su llegada a España. En esta ocasión, el equipo blaugrana jugaba en su estadio contra el Málaga, colista de la Liga, en un partido que se presumía fácil para el equipo de casa. Dos supuestos penaltis que el árbitro no sancionó en el área andaluza y un gol concedido al Málaga tras una supuesta mano, terminarán con la expulsión de Cruyff y la posterior invasión del campo por parte de los espectadores. Pese a la victoria final del Barcelona por 2-1, el público congregado en la tribuna presidencial comenzó a cantar Els Segadors, mientras la policía cargaba contra los espectadores provocando múltiples heridos y alborotos que se trasladaron a las calles que rodeaban el estadio una vez concluido el encuentro. Después de una primera sanción provisional de un partido a Cruyff, la RFEF la amplió a tres partidos. En medio de una gran polémica, el presidente del FC Barcelona, Agustí Montal, declaró días después que seguirían formando parte de la RFEF, pero que se habían equivocado cuando en su momento apoyaron a Porta como presidente, dado que "[...] no es el hombre idóneo para regir el fútbol español", al mismo tiempo que pedían su dimisión y la del presidente del Colegio de Árbitros, José Plaza (SOTERAS, 1977, p. 25). El propio Johan Cruyff, también recordaba años más tarde en su biografía cómo vivió esta situación durante los años que pasó como futbolista en el Barcelona: "Cuanto más tiempo pasaba en España, más clara tenía la importancia de la política en el juego. [...] Como jugador extranjero internacionalmente conocido, yo era intocable, de modo que podía provocar a Franco de vez en cuando." (CRUYFF, 2016, p. 80).

Por otro lado, el abuso que durante el franquismo se realizó del mito de la furia, en relación con el estilo que desde los años veinte identificaba al fútbol español (SIMÓN, 2015), provocó que según se fue asentando la nueva monarquía constitucional se irá perdiendo progresivamente esta recurrente utilización. Pese a todo, en esta primera fase de la transición, el estereotipo de la furia seguirá siendo frecuente. El énfasis en el coraje, el espíritu combativo y el valor como elementos innatos en el carácter español y, por lo tanto, en el estilo de juego de la 
selección, irá disminuyendo, mientras que por otro lado la narrativa del fracaso, el pesimismo y la mala suerte, seguirá estando estrechamente identificada con el fútbol español en los medios de comunicación.

Durante el franquismo frente a los éxitos de clubes como el Real Madrid y el FC Barcelona, la selección no consiguió clasificarse para las Copas del Mundo de 1970 y 1974, ni para la Eurocopa de 1972. Únicamente, el equipo nacional consiguió alcanzar los cuartos de final en la Eurocopa de 1976, y la clasificación para el Mundial celebrado en Argentina en 1978 y la Eurocopa de Italia en 1980. Los medios de comunicación siguieron recurriendo al discurso del fracaso nacional, a la mala suerte histórica que parecía hipotecar el futuro del deporte español. Es indudable que durante este periodo se produjo un claro desarrollo de las identidades regionales y nacionales alternativas a la española, que encontrarán en el fútbol el medio perfecto para su transmisión. La descentralización del Estado fue uno de los principales procesos que se vivieron durante estos años, como un aspecto clave en el proceso de democratización. Llopis-Coig, Martín y González Ramallal también han destacado la atención que desde estos años dedicaron los medios escritos al deporte, y en especial al fútbol, compitiendo con el inicio de la supremacía televisiva. Cabeceras como Marca, As, El Mundo Deportivo o Sport siguen hoy en día estando entre los periódicos con más lectores en España (LLOPIS-COIG; MARTíN; GONZÁLEZ RAMALLAL, 2017).

\section{A LA BÚSQUEDA DE LA MODERNIZACIÓN EN EL FÚTBOL ESPAÑOL, 1982-2004}

Los años ochenta y noventa continuaron representando para la selección española un periodo de pobres resultados, que se plasmaron a través de los medios de comunicación en un discurso, que como ya se ha mencionado en el apartado anterior, transitó reiteradamente de la reutilización del mito de la furia española y de las renovadas esperanzas de éxito, a la narración del fatalismo, la injusticia y el fracaso como elementos intrínsecos del equipo nacional. De la pobre actuación en el Mundial de 1982 a la derrota en la final de la Eurocopa de 1984, pasando por la eliminación por penaltis frente a Bélgica en la edición de 1986, sin olvidar la desafortunada derrota contra Yugoslavia en los cuartos de final del Mundial de Italia en 1990, o la enorme decepción que supuso caer eliminados frente a Italia en el Mundial de Estados Unidos cuatro años más tarde. La relación entre la selección nacional y la decepción permanente, irá en clara contraposición a los múltiples éxitos internacionales alcanzados por los clubes de fútbol españoles durante este periodo. Este último factor incidirá en la autorepresentación de un país que no terminaba de conseguir equipararse a sus vecinos europeos, tratando de buscar explicaciones en la falta de fortuna, los problemas identitarios nacionales, el miedo a la competición y al fracaso, un posible complejo inferioridad o la llegada de un excesivo número de jugadores extranjeros a la Liga española (LLOPIS-GOIG, 2015).

En relación con el Mundial de España en 1982, es interesante destacar siguiendo los estudios que Rowe ha realizado sobre el Mundial de Corea del Sur y Japón en 2002, que también se produjo una clara contradicción entre el desarrollo de nuevas audiencias globales y una imagen de internacionalismo que claramente favorecía los intereses de apertura exterior del gobierno socialista, con un discurso que a través de los medios de comunicación, pretendía utilizar este megaevento deportivo como una herramienta de nacionalización (ROWE, 2003).

Entre las elecciones electorales de 1986 y 1993, la que podríamos definir como la segunda etapa de gobierno del Partido Socialista Obrero Español (PSOE), estuvo caracterizada 
por una fase de crecimiento económico que coincidió con la integración plena de España en la Comunidad Económica Europea (CEE) en ese mismo año. En 1986 la fase de crecimiento económico tuvo su reflejo en la afluencia de capitales extranjeros, el aumento de los beneficios de las empresas, la euforia en el mercado financiero, o el enorme auge inmobiliario con subidas de precios sin precedentes hasta ese momento.

Los años noventa marcaron el inicio del agotamiento del programa socialista de 1982, pese que el esfuerzo inversor del Estado, favorecido por el impulso de los grandes eventos internacionales que albergó el país en esos años -los Juegos Olímpicos y la Exposición Internacional de Sevilla, ambos en 1992-, permitió la modernización de las deterioradas y obsoletas infraestructuras del país. Pero el escenario económico se vio modificado radicalmente en septiembre de 1992, cuando se inició una fase de crisis, desempleo e inflación a la que se unió el mencionado declive del PSOE. Las elecciones generales de marzo 1996 dieron el triunfo por un escaso margen a los conservadores del Partido Popular, aprovechando, entre otros factores, el desprestigio socialista durante su último mandato.

EI PSOE trató de vincularse a la selección española en varias ocasiones, como por ejemplo en diciembre de 1983, en ocasión de la victoria frente a la selección de Malta por 12-1 en un encuentro valedero para la clasificación de la Eurocopa de Francia 84; o en junio de 1986, pocos días antes de las elecciones generales cuando en el Telediario del primer canal de TVE, se emitieron sobrepuestas las siglas de "PSOE" con el resumen de la goleada de España a Dinamarca por 5-1 durante el Mundial de México (EL RÓTULO, 1986).

En las elecciones de 1996 el Partido Popular (PP) se impondrá al PSOE, aunque no logró la mayoría absoluta en el Parlamento, obligándoles a tener que pactar con la derecha nacionalista catalana y vasca. El PP implementó una política de corte neoliberal y de privatización de un buen número de empresas que aun pertenecían al Estado; al mismo tiempo que se incidió en impulsar un proceso nacionalizador en el que se compartía un discurso sobre el concepto de España que mezclaba aspectos tradicionales e históricos, con la imagen de un nuevo país moderno que buscaba aumentar su protagonismo en Europa. Al mismo tiempo, el fútbol experimentó una gran transformación durante las dos últimas décadas, influido por el inicio de una fase de globalización y mercantilización en el que la liberalización del mercado audiovisual y los derechos de retransmisión televisiva de los encuentros de fútbol se convirtieron en factores claves. Durante este periodo la selección española y el Real Madrid perdieron el rol hegemónico que habían protagonizado durante el franquismo, y empezó a mostrarse a través de la prensa una España multinacional y compleja que encontró en los estadios su lugar de expresión (LLOPIS-GOIG, 2015).

El recurso a la furia dejó progresivamente de funcionar en las narraciones deportivas durante los años ochenta y noventa, aunque aparecerá en ocasión de triunfos concretos como la victoria de la medalla de oro del equipo de fútbol masculino en los Juegos Olímpicos de Barcelona, o el mencionado triunfo frente a Malta en 1983. En cambio, el estereotipo del fracaso siguió estando plenamente integrado en la identidad nacional. La derrota de España frente a Francia en la final de la Eurocopa de 1984, con una desgraciada intervención del portero, y la eliminación en cuartos de final del Mundial de Estados Unidos en 1994, al perder frente a Italia en un dramático partido, elevaron al máximo los niveles de fatalismo convirtiéndose, de alguna forma, en el reflejo de la debilidad de un Estado-nación (PÉREZ DE ROZAS, 1984). 


\title{
4 DE JOSÉ LUIS RODRÍGUEZ ZAPATERO A MARIANO RAJOY: LA NARRATIVA DEL ÉXITO Y LA “FUTBOLIZACIÓN” DEL PROBLEMA IDENTITARIO CATALÁN, 2004-2017
}

\begin{abstract}
A veces la realidad es más grande que los sueños. Habéis demostrado que con talento, trabajo, esfuerzo y humildad se puede alcanzar el éxito y éste ha llegado con un juego espectacular y brillante. No sólo ha ganado España, también el fútbol en general porque lo habéis hecho grande (GABILONDO, 2008, 2 jul.).
\end{abstract}

Las palabras pronunciadas por el expresidente José Luis Rodríguez Zapatero en la recepción que ofreció en el Palacio de la Moncloa al equipo nacional después de su victoria en la Eurocopa de 2008, representan el inicio de un cambio de ciclo que con los posteriores triunfos en el Mundial de Sudáfrica en el 2010 y la Eurocopa del 2012, han marcado los mayores éxitos en la historia del fútbol español. A partir de este momento, el discurso de la furia y el fracaso ha dado paso a lo que podríamos denominar como la "narrativa del éxito". De un fútbol caracterizado por elementos como el pundonor y la lucha, con pocas concesiones a las habilidades técnicas, se evolucionó desde la segunda mitad de los años noventa a un juego de posesión donde primaba el elemento técnico a lo físico, con un estilo de juego espectacular y brillante. Los elementos de comunicación han representado un papel determinante en la transformación desde los últimos años del discurso identitario español relacionado con el fútbol, así como en la construcción y fortalecimiento de las propias identidades nacionales como ya habían demostrado autores como Anderson (1983), Hobsbawm y Ranger (1983).

Al mismo tiempo, tanto los éxitos de la selección y los clubes de fútbol, como del deporte español en general, han servido de amortiguador de las tensiones de un país que desde la segunda legislatura de Zapatero (2008-2011), se ha visto inmerso en una profunda crisis económica. El gobierno no dudó en vincularse directamente con los éxitos del deporte español. El propio presidente afirmó que, con la victoria en la Eurocopa de Austria y Suiza, se había concluido "[...] la transición del fútbol español" (MÉNDEZ, 2008). Como defiende Quiroga (2014, p. 246), excepto en el fútbol, los medios de comunicación europeos han vuelto en los últimos años a seguir representando a los españoles utilizando los tradicionales estereotipos de desorganizados, holgazanes y derrochadores. Esta simplificación de la realidad sólo pareció modificarse cuando se elogiaban los triunfos del fútbol español en la cobertura futbolística de estos medios.

El proceso de "deportivización" que vivieron los medios de comunicación desde la primera década del siglo XXI favoreció el refuerzo de las nociones de identidad asociadas al Estado-nación. Si por un lado el fútbol ha seguido permitiendo la recuperación de la identidad patriótica asociada al equipo nacional, es también cierto que durante estos últimos años se ha producido un fuerte impulso de lo que podíamos denominar nacionalismos subestatales. Clubes como el FC Barcelona, el Athletic de Bilbao o la Real Sociedad han ayudado a la difusión de narrativas nacionalistas. La llegada de Joan Laporta a la presidencia del FC Barcelona en el 2003, marcó el inicio de un proceso de re-catalanización del club estableciendo un fuerte paralelismo entre el club y Cataluña. Los triunfos y el juego espectacular del equipo a partir de la llegada de Pep Guardiola a la dirección técnica en el 2008, provocó que al mismo tiempo que se acentuaba el discurso nacionalista el FC Barcelona se convirtiera en el equipo favorito de los españoles (NIETO, 2011). A este proceso se añadió el importante papel que tuvieron en los éxitos de la selección los jugadores del FC Barcelona.

Durante los últimos años ha aumentado el apoyo a un Estado independiente desde ciertos sectores de la sociedad catalana. Al mismo tiempo, las victorias de la selección 
española entre el 2008 y el 2012 han mostrado un fenómeno interesante que incide en la diversidad de identidades que hoy en día conviven en la sociedad catalana. El 10 de julio del 2010, cerca de 425.000 personas tomaron las calles de Barcelona para protestar por el fallo del Tribunal Constitucional que avalaba la inclusión del término nación en el Preámbulo del nuevo Estatuto de Autonomía, pero que carecía de eficacia jurídica interpretativa. El día después, el Ayuntamiento de Barcelona mencionó que 75.000 personas habían presenciado la final del Mundial de Sudáfrica a través de la pantalla gigante que se había instalado en el centro de la ciudad (CORDOVILLA, 2010). Estos ejemplos muestran de forma patente la pluralidad de identidades nacionales que existen hoy en día en Cataluña. Al mismo tiempo, y como ha demostrado González Ramallal a través de su estudio sobre la cobertura informativa de la selección española durante el Mundial de Sudáfrica, la prensa deportiva se utilizó durante la duración de este evento como una herramienta para el fortalecimiento de identidades españolas y catalanas (GONZÁLEZ RAMALLAL, 2014)

En relación con el conflicto identitario en Cataluña, que ha alcanzado sus cotas más altas de tensión social durante los últimos meses del 2017, el fútbol ha representado un papel de gran importancia política. Aprovechando la disputa del "clásico", entre el FC Barcelona y el Real Madrid en octubre del 2012, la directiva del club catalán decidió crear un gran mosaico compuesto de 98.000 cartulinas que rodeó todo el Camp Nou con una gran senyera, mostrando la inscripción de "Barça", igualmente, en el calentamiento se colgó una pancarta en inglés con el lema "Cataluña, nuevo Estado de Europa" y parte del estadio gritó "in-inde-independència" (PIÑOL, 2012).

Pocos días antes de que se celebrasen las elecciones catalanas de septiembre del 2015, presentadas por los propios partidos independentistas como un plebiscito a favor o en contra de la independencia de Cataluña, el presidente del CSD Miguel Cardenal, afirmaba que cuestionaba la capacidad del FC Barcelona para generar ingresos económicos en el marco de una Cataluña independiente (CARDENAL, 2015). En septiembre de ese mismo año, se presentó la campaña \#Guanyarem, en la que se recogían los testimonios de diferentes personalidades del deporte catalán. Xavier Vinyals, presidente de la Plataforma Pro Selecciones Catalanas, aseguró que "algunos deportistas tienen miedo a significarse políticamente y poner en riesgo sus carreras profesionales", aunque destacaba que dicha campaña "es transversal y no está asociada a ningún partido político" (GIRALDO, 2015).

En este análisis, es necesario incidir en la gran polémica que despertó el posicionamiento político del jugador catalán Gerard Piqué, defendiendo en los medios de comunicación y en las redes sociales el derecho de los ciudadanos catalanes a decidir su relación con el Estado español mediante un referéndum. El jugador aseguró que formar parte de la selección española no era contradictorio con defender la consulta popular: "[...] yo me siento muy comprometido y muy a gusto. Siempre que venga con la selección estaré encantado. Soy futbolista, pero primero de todo soy ciudadano y cuando siento que debo involucrarme, lo hago" (PIQUÉ, 2016). Las declaraciones del jugador han provocado, a partir de ese momento, que su presencia con la selección española haya estado acompañada de reiterados silbidos y abucheos por parte de los aficionados tanto en partidos como entrenamientos.

Las encuestas realizadas por el Centre d'Estudis d'Opinió (CEO) a principios del 2016, cifraban en menos del 40 por ciento el número de independentistas, mientras quienes sólo se reconocían catalanes, pasaron del 14,9 por ciento en junio del 2005, al 26,3 por ciento 
en marzo del 2016 (MONREAL; ARAGÓ, 2016). La celebración de la consulta popular no reconocida por el gobierno el 1 de octubre del 2017, volvió a mostrar el papel que representa el fútbol en la construcción identitaria en este país. Pep Guardiola, al igual que Gerard Piqué, también se ha implicado de forma muy activa en la campaña a favor de la consulta. El actual entrenador del Manchester City, mostró en un vídeo de la Asamblea Nacional Catalana (ANC) su apoyo al referéndum: "Esto no va de independencia, va del derecho a decidir poder votar. Esto no va de independencia, va de democracia" (PEP, 2017).

El FC Barcelona también se posicionó a favor del referéndum y el derecho de Cataluña a decidir. El comunicado destacaba que el club "[...] desde el máximo respeto a la pluralidad de su masa social, seguirá apoyando la voluntad de la mayoría del pueblo de Cataluña, expresada siempre de una forma cívica, pacífica y ejemplar" (EL BARCELONA, 2017). El 1 de octubre, día en el que se realizó la consulta popular, el FC Barcelona debía disputar su encuentro de Liga frente a la UD Las Palmas. El partido se disputó finalmente a puerta cerrada. El propio presidente del Barcelona, declaró poco antes del inicio del encuentro que "[...] ante la situación excepcional que se ha vivido en Cataluña", en clara referencia a los altercados que se habían producido entre algunos ciudadanos y las fuerzas de seguridad, se había tratado con la LFP para que se aplazara el encuentro. Al ser imposible, se tomó la decisión de jugar a puerta cerrada, Bartomeu seguirá mencionando que con esta decisión "hemos querido de esta forma que el mundo entero vea cómo estamos sufriendo y cuál es la situación en Cataluña", y añadía que "el Barça siempre ha estado con la libertad de expresión la democracia y el derecho a decidir. Son valores que históricamente ha defendido el Barça y haremos acciones. El Barça ha de estar con la gente" (TRONCHONI, 2017)

Esta decisión provocó la fractura en el seno de la directiva, al dimitir el vicepresidente del Área de Relaciones Internacionales e Institucionales, Carles Vilarrubí, y el directivo Jordi Monés. Al final del encuentro, Piqué criticó la decisión del gobierno de España de impedir el voto, señalando que "durante muchos años no se pudo votar en este país y es un derecho que debemos defender. Soy y me siento catalán y hoy más que nunca me siento orgulloso. La gente se ha comportado de forma extraordinaria". A continuación, entre lágrimas, mencionó que "[...] no ha habido ningún acto de agresión y ha tenido que venir la Policía Nacional y la Guardia Civil para actuar de la forma que han actuado" (FONT, 2017, p. 67). Mientras tanto, ese mismo día en el Santiago Bernabéu, los aficionados del equipo madrileño recibían a su equipo frente al RCD Español con las gradas llenas de banderas españolas. $A B C$ describió este hecho, como "si algunos, traumatizados por lo visto en Cataluña durante la jornada, quisieran refugiar la nación en el poderoso recinto del Madrid" (HUGHES, 2017, p. 68).

\section{CONCLUSIONES}

A partir de la muerte del general Franco el fútbol, al igual que la sociedad española, se embarcó en un intenso proceso de democratización. Mientras tanto, los nacionalismos periféricos encuentran nuevamente en el fútbol un catalizador de sus sueños identitarios. Eventos como el Mundial de España en 1982 y los Juegos Olímpicos diez años después, intentaban mostrar al mundo un país moderno y democrático preparado para integrarse en Europa y que había dejado olvidados en el pasado los oscuros años del franquismo. Pese a todo, la narrativa de la furia y el fracaso siguió volviendo a las portadas de los principales medios de comunicación 
en ocasión de las grandes citas internacionales. La imagen negativa, de un país que afrontaba la mayor crisis económica de su historia, estaba sirviendo para renovar los viejos estereotipos referidos a los españoles. Esta dinámica sólo cambiará con el ciclo de triunfos que se iniciaron con la Eurocopa del 2008, y tuvieron su continuidad con las victorias en el Mundial del 2010 y nuevamente en la Eurocopa del 2012 (Polonia-Ucrania). El éxito deportivo, permitió mostrar al exterior una imagen de éxito y modernidad muy alejada de la realidad económica y social que vivía el país. Al mismo tiempo, y frente a la imagen de unidad nacional que mostraba el fútbol y que difundían las televisiones internacionales, existían profundos conflictos identitarios que el enquistamiento de la crisis económica terminó sacando a la luz.

Durante el franquismo, la prensa utilizó de forma reiterada el fútbol como un medio de nacionalización española de gran efectividad. Aunque en la posterior fase de transición democrática podría parecer que se atenuó dicha función, este estudio ha podido demostrar cómo estos medios de comunicación siguieron instrumentalizando de forma reiterada el fútbol, pese a que los éxitos de la selección española fueron irrelevantes. La rivalidad entre clubes, principalmente FC Barcelona y Real Madrid, ha sido también utilizada por los medios de comunicación como creadores de identidades simbólicas, el enfrentamiento "nosotros" y "ellos" en función de unos estereotipos construidos gracias a un aumento exponencial de la cobertura futbolística en la prensa, y en gran medida centrada en la información relacionada con estos dos clubes. El relato nacional-nacionalista catalán ha encontrado en el FC Barcelona el medio ideal con el que poder mostrar su enfrentamiento con el Gobierno español, ocupando un importante espacio en los medios de comunicación nacionales e internacionales. Al mismo tiempo, los triunfos del Real Madrid y de la selección española también han sido objeto de apropiación política por parte de determinados sectores, retroalimentando el juego de enfrentamiento político-deportivo y cohesionando a través del fútbol el relato del nacionalismo español.

Como ha podido comprobarse en esta investigación, el fútbol como aglutinador de pasiones, hecho social total y herramienta de construcción identitaria, ha sido utilizado recurrentemente desde la transición por los principales actores políticos y los medios de comunicación. Especialmente en los últimos años, con el enquistamiento de la "cuestión catalana" y la deriva independentista, junto con la potenciación de los estereotipos políticos que acompañan desde su origen al FC Barcelona y al Real Madrid, se ha podido observar cómo el fútbol sigue utilizándose como potenciador de los discursos identitarios en la sociedad, con los medios de comunicación como su principal altavoz pese a la simplicidad de su discurso.

El fútbol, de la mano de la prensa, se ha convertido desde el inicio de la transición democrática en un factor de gran importancia para construir y transformar los relatos identitarios en España. Pese a que los discursos y planteamientos políticos que encontramos en estos medios no son muy elaborados, sería una equivocación el despreciar la importancia que representa el fútbol como elemento de estudio desde la historia y las ciencias sociales. En concreto, es necesario seguir investigando sobre cómo el fútbol a través de los medios de comunicación se convierte en una herramienta de gran efectividad como agente socializador de nuevos discursos identitarios. Al mismo tiempo, es de gran interés el comprobar que clubes como el FC Barcelona y el Real Madrid representan de forma exponencial lo que podríamos denominar como "fútbol global", con miles de aficionados por todo el planeta, estrategias de mercado a nivel global y jugadores de múltiples nacionalidades, al mismo tiempo que en estos equipos cada día son más potentes la construcción de relatos identitarios desde una perspectiva 
local, en donde el significado territorial y los vínculos con el entorno geográfico cohesionan a los aficionados de una misma ciudad o región.

Es difícil poder prever el desenlace de la aventura secesionista que se ha iniciado en los últimos años, pero todo parece indicar que la reconstrucción de las roturas sociales llevará mucho tiempo tanto para Cataluña como para España. Un hipotético escenario de independencia plantea desde una perspectiva futbolística muchas incógnitas, que abarcan desde el rol que tendrían clubes catalanes como el FC Barcelona y el Espanyol en la Liga española, hasta la posible participación o no de futbolistas catalanes en la selección, y la propia cuestión de la creación de una liga catalana. Lo que sí que parece difícil que se pueda cambiar en los próximos años, es la reiterada utilización del fútbol como un instrumento para la creación y transmisión de relatos identitarios catalanes y españoles.

\section{REFERENCIAS}

ANDERSON, Benedict. Comunidades imaginadas: Reflexiones sobre el origen y la difusión del nacionalismo. México D. F.: Fondo de Cultura Económica, 1983.

BODIN, Dominique. Inclusión social y práctica deportiva. El deporte como herramienta de construcción ciudadana en la España democrática, 1975-2000. En: PUJADAS, Xavier. (Coord.). Atletas y ciudadanos: Historia social del deporte en España, 1870-2010. Madrid: Alianza, 2011. p. 433-466.

BOLETÍN OFICIAL DEL ESTADO [BOE España], sec. 1, n. 260, p. 103529-103544, 27 oct. 2017. Disponible en: < https://www.boe.es/boe/dias/2017/10/27/pdfs/BOE-A-2017-12328.pdf>. Acceso en: 24 oct. 2018.

CARDENAL cree que con una Catalunya independiente el Barça no pasaría de cuartos en la Champions. Sport, Barcelona, 21 sept. 2015. Disponible en: <https://www.sport.es/es/ noticias/barca/cardenal-cree-que-con-una-catalunya-independiente-barca-no-pasaria-cuartoschampions-4525855> Acceso en: 210ct. 2018.

CORDOVILLA, Anna. España se echa a la calle. Mundo Deportivo, Barcelona, p. 30, 12 jul. 2010.

CRUYFF, Johan. 14. La autobiografía. Barcelona: Planeta, 2016.

EL RÓTULO del PSOE apareció sobre la imagen de un gol de Butragueño. Diario 16, Madrid, 20 jun. 1986.

EL BARCELONA se posiciona sobre el 1-0 "en defensa del país" y pide "libertad" para votar.

El Confidencial, Madrid, 20 sept. 2017. Disponible en: <https://www.elconfidencial.com/deportes/ futbol/2017-09-20/el-barcelona-se-posiciona-sobre-el-1-0 1446829/> Acceso en: 24 oct. 2018.

FONT, S. Ir a la selección no es una cuestión de patriotismo. ABC, Madrid, p. 67, 2 oct. 2017.

GABILONDO, Aritz. Zapatero: no sólo ganó España, ganó el fútbol. Diario As, Madrid, 2 jul. 2008. Disponible en: <https://as.com/futbol/2008/07/02/mas futbol/1214980048 850215 html> Acceso en: 24 nov. 2018. 
GALLO, J. Intolerable orgía separatista. El Alcazar. Madrid, 7 dic. 1976. Disponible en: <https:// linz.march.es/documento.asp?reg=r-38804> Acceso en: 20 oct. 2018.

GARCÍA MARTÍ, Carlos. Reshaping Spanish Football Identity in the 1940s: From Fury to Tactics. The International Journal of the History of Sport, v. 33, n. 10, p. 1116-1132, 2016.

GIRALDO, Javier. Bartomeu y Collet, con la campaña \#Guanyarem del Deporte catalán. Sport, Barcelona, 7 sept. 2015. Disponible en: <https:/www.sport.es/es/noticias/barca/bartomeu-colletcon-campana-guanyarem-deporte-catalan-4486994> Acceso en: 20 sept. 2018.

GONZÁLEZ RAMALLAL, Manuel E. Prensa deportiva e identidad nacional: España en el Mundial de fútbol de Sudáfrica 2010. Política y Sociedad, v. 51, n. 2, p. 337-366, 2014.

HOBSBAWM, Eric; RANGER, Terence. The Invention of Tradition. Cambridge: Cambridge University Press, 1983.

HUGHES. Isco añade goles a su recital. ABC, Madrid, p. 68, 2 oct. 2017.

LLOPIS-GOIG, Ramón. Spanish Football and Social Change: Sociological Investigations. London: Palgrave Macmillan, 2015.

LLOPIS-COIG, Ramón; MARTíN, Montse; GONZÁLEZ RAMALLAL, Manuel. Medios de comunicación, deporte y sociedad. En: GARCÍA FERRANDO, Manuel et al. (Eds.). Sociología del deporte. Madrid: Alianza, 2017. p. 197-222.

MATEO, J. J. Rajoy destituye a Puigdemont y convoca elecciones en Cataluña el 21 de diciembre. El País, Madrid, 28 oct. 2017. Disponible en: <https://elpais.com/politica/2017/10/27/ actualidad/1509114362_109606.html> Acceso en: 20 sept. 2018.

MÉNDEZ, Rafael. Zapatero: "Se ha cerrado la transición del fútbol español". El País. Madrid, 1․ jul. 2008. Disponible en: <https://elpais.com/diario/2008/07/01/deportes/1214863205 850215.html> Acceso en: 21 oct. 2018.

NIETO, Luis. España se pasa del Madrid al Barcelona. Diario As, Madrid, 10 oct. 2011. Disponible en: <https://as.com/futbol/2011/10/10/mas futbol/1318228009 850215.html> Acceso en: 21 oct. 2018.

PEP Guardiola apoya el referéndum en un vídeo de la ANC. ABC, Madrid, 24 sept. 2017. Disponible en: <https://www.abc.es/deportes/futbol/abci-guardiola-apoya-referendumvideo-201709241020 noticia.html> Acceso en: 21oct. 2018.

PÉREZ DE ROZAS, Emilio. Los franceses heredaron la fortuna de los españoles. EI País. Madrid, 28 jun. 1984. Disponible en: <https://elpais.com/diario/1984/06/28/ deportes/457221601 850215.html> Acceso en: 23 mar. 2018.

PIÑOLS, Àngels. Clamor soberanista en el minuto 17. El País. Madrid, 1ํ. oct. 2012. Disponible en: <https://elpais.com/deportes/2012/10/07/actualidad/1349640813 429916.html> Acceso en: 20 oct. 2018.

PIQUÉ: "Estoy a favor de la consulta, no de la independencia de Cataluña". La Información, Madrid, 16, febr. 2016. Disponible en: <https://www.lainformacion.com/deporte/futbol/pique-estoya-favor-de-la-consulta-no-de-la-independencia-de-cataluna jiFriMy4mqxzxcwWx7UJj1/> Acceso en: 21 sept. 2017.

PUIGDEMONT, Carles. [@KRLS]. La victòria del @ GironaFC sobre un dels grans equips del món és tot un exemple i un referent per a moltes situacions. Twitter, 29 oct. 2017. 
Disponible en: <https://twitter.com/KRLS/status/924697593468375040?ref src=twsrc\%5Etfw\&ref url=http\%3A\%2F\%2Fwww.laopinioncoruna.es\%2Fespana\%2F2017\%2F10\%2F29\%2Fpuigdemontcompara-girona-victoria-madrid\%2F1230780.html> Acceso: 06 dic. 2017.

QUIROGA FERNÁNDEZ DE SOTO, Alejandro. Goles y banderas: Fútbol e identidades nacionales en España. Madrid: Marcial Pons, 2014.

RODRIGÁLVAREZ, Eduardo. Sacar la ikurriña fue una decisión unánime. El País, Madrid, 22 abr. 2011.

ROWE, David. Global Media Sport: Flows, Forms and Futures. Londres: Bloomsbury, 2011.

SANTANDER: el público obliga a retirar una 'ikurriña' en un partido de fútbol. ABC, Madrid, p.6, 20 nov. 1979.

SIMÓN, Juan Antonio. Construyendo una pasión: el fútbol en España, 1900-1936. La Rioja: UNIR, 2015.

SOTERAS, Alfonso. Dimisión de Porta y Plaza. Informaciones, Madrid, p. 25, 16 febr. 1977.

SOTERAS, Alfonso. El encuentro de dos viejos pueblos. La Vanguardia, Barcelona, p. 47, 22 febr. 1977.

TEBAS MEDRANO, Javier. [@Tebasjavier]. Hoy estuvimos en la plaza Colón, mañana en Barcelona, plaza Urquinaona a las 12h. Porque Cataluña es España. Twitter, 7 oct. 2017. Disponible en: <https://twitter.com/Tebasjavier/ status/916664242518069248? ref src=twsrc\%5Etfw\&ref url=http\%3A\%2F\%2Fwww.marca. com\%2Ffutbol\%2F2017\%2F10\%2F08\%2F59da5703e2704e4a208b45ca.html> Acceso en: 06 dic. 2017.

TRONCHONI, Nadia. Bartomeu: "A puerta cerrada para que el mundo entero vea cómo sufrimos en Cataluña". El País, Madrid, 1‥ oct. 2017. Disponible en: <https://elpais.com/ deportes/2017/10/01/actualidad/1506868240 917498.html> Acceso en: 19 Sept. 2018. 
